\title{
Metaanalysis of ${ }^{68}$ Ga-PSMA-11 PET Accuracy for the Detection of Prostate Cancer Validated by Histopathology
}

\author{
Thomas A. Hope ${ }^{1-4}$, Jeremy Z. Goodman ${ }^{4}$, Isabel E. Allen ${ }^{5}$, Jeremie Calais ${ }^{6}$, Wolfgang P. Fendler ${ }^{7}$, and \\ Peter R. Carroll ${ }^{3,4}$ \\ ${ }^{I}$ Department of Radiology and Biomedical Imaging, UCSF, San Francisco, California; ${ }^{2}$ Department of Radiology, San Francisco VA \\ Medical Center, San Francisco, California; ${ }^{3}$ UCSF Helen Diller Family Comprehensive Cancer Center, San Francisco, California; \\ ${ }^{4}$ Department of Urology, UCSF, San Francisco, California; ${ }^{5}$ Department of Epidemiology and Biostatistics, UCSF, San Francisco, \\ California; ' Ahmanson Translational Imaging Division, Department of Molecular and Medical Pharmacology, UCLA, Los Angeles, \\ California; and ${ }^{7}$ Department of Nuclear Medicine, University Hospital Essen, Essen, Germany
}

\begin{abstract}
${ }^{68} \mathrm{Ga}-\mathrm{PSMA}-11 \mathrm{PET}$ is used to stage patients with prostate cancer. We performed an updated metaanalysis that separates imaging at the time of diagnosis and at the time of biochemical recurrence and focuses on pathology correlation in both populations. Methods: We searched the MEDLINE and EMBASE databases using the PRISMA statement. Quality was assessed using the Quality Assessment of Diagnostic Accuracy Studies tool 2. In total, 1,811 studies were screened, 58 were analyzed, 41 were included for qualitative synthesis, and 29 were included for quantitative analysis. A random-effect model and a hierarchical summary receiver-operating-characteristic model were used to summarize the sensitivity, specificity, positive predictive value (PPV), negative predictive value, and accuracy for pelvic lymph nodes in initial staging compared with pathology at prostatectomy and the PPV for lesions with pathologic correlation in those with biochemical recurrence. We also summarized the detection rate of ${ }^{68} \mathrm{Ga}-\mathrm{PSMA}-11$ in those with biochemical recurrence stratified by prostate-specific antigen (PSA) at the time of imaging. Results: The metaanalysis of ${ }^{68} \mathrm{Ga}-\mathrm{PSMA}-11$ at initial staging demonstrated a sensitivity and specificity of 0.74 (95\% confidence interval [95\% Cl], 0.51-0.89) and $0.96(95 \% \mathrm{Cl}, 0.85-0.99)$, respectively, using nodal pathology at prostatectomy as a gold standard. At biochemical recurrence, the PPV was $0.99(95 \% \mathrm{Cl}$, $0.96-1.00)$. The detection rate was $0.63(95 \% \mathrm{Cl}, 0.55-0.70)$, with a PSA of less than 2.0 and $0.94(95 \% \mathrm{Cl}, 0.91-0.96)$ with a PSA of more than 2.0. Conclusion: ${ }^{68} \mathrm{Ga}-\mathrm{PSMA}-11$ performed well for the localization of metastatic prostate cancer at initial staging and at the time of biochemical recurrence.
\end{abstract}

Key Words: molecular imaging; oncology; GU; PET; PSMA; prostate cancer

J Nucl Med 2019; 60:786-793

DOI: 10.2967/jnumed.118.219501

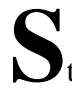
of patients with prostate cancer using conventional imaging, typically MRI, CT, and bone scans, is limited by a low sensitivity for metastatic disease. Imaging using small molecules

Received Aug. 21, 2018; revision accepted Nov. 1, 2018.

For correspondence or reprints contact: Thomas A. Hope, Department of Radiology and Biomedical Imaging, University of California, San Francisco, 505 Parnassus Ave., M-391, San Francisco, CA 94143.

E-mail: thomas.hope@ucsf.edu

Published online Dec. 7, 2018.

COPYRIGHT (C 2019 by the Society of Nuclear Medicine and Molecular Imaging. targeting the prostate-specific membrane antigen (PSMA) has demonstrated higher detection sensitivity than conventional imaging and other radiotracers such a choline-based agents (1-3). Although there is a large number of radiotracers that target PSMA, ${ }^{68} \mathrm{Ga}$-PSMA-11 constitutes most of the literature.

Paralleling its widespread clinical adoption, a large number of publications on ${ }^{68} \mathrm{Ga}$-PSMA-11 PET emerged over the past $4 \mathrm{y}$. Several metaanalyses have been performed. However, prior metaanalyses are limited by the heterogeneity of included studies: patients at initial diagnosis and at biochemical recurrence were combined, and the reference standard for lesion validation ranged from clinical experience and imaging without predefined criteria to surgery or biopsy in a few cases $(4,5)$. However, to achieve the approval of PSMA ligands for PET imaging, systematic analysis of categorized evidence using histopathology validation is needed. Additionally, since the publication of initial metaanalyses, nearly 3 times as many patients have been reported in the literature.

We therefore focused on the 2 indications for which ${ }^{68} \mathrm{Ga}-$ PSMA-11 PET is most likely to be used clinically: initial staging of those with intermediate- to high-risk prostate cancer and localization of metastatic disease in those with biochemical recurrence after definitive therapy. We performed an updated metaanalysis, separating such patients and correlating findings with pathologic validation. We specifically focused on lesions with pathologic validation to support the potential approval of this drug.

\section{MATERIALS AND METHODS}

\section{Search Strategy}

The aim of this systematic review and metaanalysis was to summarize studies of staging and restaging ${ }^{68} \mathrm{Ga}$-PSMA PET/CT or PET/MRI in patients with either localized or metastatic prostate cancer. A second aim was to determine the imaging test accuracy of the new PET/CT and PET/MRI method using tissue samples obtained through biopsy or surgery as the reference standard. We followed the PRISMA guidelines (6). The protocol for this metaanalysis was registered with PROSPERO (Centre for Reviews and Dissemination temporary identification number 99828).

The scientific literature databases MEDLINE and EMBASE were systematically searched in April 2018. Our systematic review included original research studies of initial staging and biochemical recurrence patients with ${ }^{68} \mathrm{Ga}$-PSMA-11 PET. The search used several key words, including "prostate" or "prostate cancer" or "prostate neoplasm" or "prostate malignancy"; "positron emission tomography" or "PET"; and "prostate specific membrane antigen" or "PSMA" and "PSMA 
PET" with "prostate." The search and article selection were performed by 2 independent evaluators. Each screened the titles and abstracts of the reports and selected appropriate original research articles that were published in English. Papers that were excluded included those published before 2012, studies of laboratory results, studies of neoplasms other than prostate cancer, studies of radiotracers that were not ${ }^{68} \mathrm{Ga}-$ PSMA-11, bioavailability studies, case studies, and studies with small sample sizes ( $<20$ patients). Risk for bias in the studies was assessed using the QUADAS-2 methodology (7).

\section{Outcome Measures}

We looked at several outcome measurements from the papers reviewed. We calculated imaging test characteristics (sensitivity, specificity, positive predictive value (PPV), negative predictive value (NPV), and accuracy) for the detection of lesions in the prostate and pelvic lymph nodes and compared those values with the results of histopathologic biopsy or radical prostatectomy lymph node dissection. Sensitivity is defined as true-positives divided by true-positives plus false-negatives. Specificity is defined as true-negatives divided by true-negatives plus false-positives. PPV is defined as true-positives divided by true-positives plus false-positives. NPV is defined as true-negatives divided by truenegatives plus false-negatives. Accuracy is defined as true-positives plus false-positives divided by the population. We also calculated detection rate in patients by prostate-specific antigen (PSA) cutoffs that had positive imaging results by ${ }^{68} \mathrm{Ga}-\mathrm{PSMA}-11$. Detection rate is defined as the percentage of patients who have focal uptake on ${ }^{68} \mathrm{Ga}$-PSMA11 PET that is interpreted as being consistent with cancer.

\section{Data Collection}

Two reviewers independently extracted from the studies the radiation dose and uptake time for ${ }^{68} \mathrm{Ga}$-PSMA PET/CT and PET/MRI. The reviewers also extracted the number of patients in each study, their age (median and mean), PSA (median and range), Gleason score, and initial treatment (androgen deprivation therapy, radiation therapy, or radical prostatectomy). The number of patients detected with PET imaging and the location of the metastases were also extracted.

\section{Metaanalytic Methods}

In our metaanalysis, we used a random-effect model (8) and a hierarchical summary receiver-operating-characteristic model (Stata, version 14.0; StataCorp). We summarized the sensitivity, specificity, PPV, NPV, and accuracy for pelvic lymph nodes in initial staging using pathology at prostatectomy as a gold standard, and for any lesion with pathologic correlation in biochemical recurrence patients. We also summarized the detection rate of ${ }^{68} \mathrm{Ga}$-PSMA-11 in those with biochemical recurrence stratified by PSA at the time of imaging. All point estimates from the metaanalysis regression are reported as the mean and $95 \%$ confidence interval $(95 \% \mathrm{CI})$.

\section{RESULTS}

\section{Eligible Studies}

Electronic searching of PubMed and EMBASE resulted in 2,178 articles (Fig. 1). Of these, 367 were duplicates, and 1,811 were reviewed at the abstract level, of which 1,763 were excluded. Subsequently, 59 papers were reviewed in full text, and 18 studies were excluded. Forty-one articles were deemed eligible for inclusion in the metaanalysis (Table 1). Nearly all papers imaged patients roughly $60 \mathrm{~min}$ after injection with a dose of 120-230 MBq. Risk for bias and applicability were assessed using the QUADAS2 tool (Supplemental Fig. 1; supplemental materials are available at http://jnm.snmjournals.org). Significant biases existed in most papers reviewed. Bias concerned the selection of patients, as nearly all studies reported retrospective cohorts without predefined inclusion criteria, and lack of a histologic reference standard, with, again, most papers reporting only detection rates and only a few reporting results compared with histology. Overall, the performance of ${ }^{68} \mathrm{Ga}$-PSMA-11 PET was fairly consistent across papers, with uptake times and doses in similar ranges. Most of the included papers were retrospective studies that had patients not enrolled on the basis of defined inclusion criteria, and only 4 of the studies were prospectively acquired (Table 1).

\section{Initial Staging}

Six studies in the metaanalysis included patients imaged at initial staging, of which 5 papers correlated pelvic nodal disease to pathology at radical prostatectomy, reporting a total of 266 patients between the 5 studies (Table 2). Most of the papers included only patients who were classified as at intermediate to high risk by the D'Amico classification (Table 2). Across the 5 studies, the metaanalysis of the sensitivity, specificity, PPV, NPV, and accuracy was 0.74 (95\% CI, 0.51-0.89), 0.96 (95\% CI, $0.85-0.99), 0.93$ (95\% CI, 0.86-0.99), 0.85 (95\% CI, 0.75-0.93), and 0.86 (95\% CI, 0.79-0.92), respectively (Fig. 1).

\section{Biochemical Recurrence}

Thirty-four studies in the metaanalysis included patients imaged at biochemical recurrence. First, we reviewed all included papers to determine whether results were reported using pathology as a gold standard and selected all patients for whom a pathology correlation was reported. Most papers on patients with biochemical recurrence did not have pathologic correlation for PSMA-avid lesions, and only detection rates were reported. In total, 256 patients were included across 15 studies with pathologic correlation, of which 233 were reported as truepositive lesions (Table 3 ). The metaanalysis of the sensitivity, specificity, PPV, NPV, and

FIGURE 1. PRISMA flow diagram depicting process for selecting papers included in this metaanalysis. 
TABLE 1

Characteristics of Studies Included

\begin{tabular}{|c|c|c|c|c|c|c|c|c|c|}
\hline \multirow[b]{2}{*}{ Study } & \multirow[b]{2}{*}{ Year } & \multirow[b]{2}{*}{ Patients $(n)$} & \multirow[b]{2}{*}{ Indication } & \multirow[b]{2}{*}{ Age (y) } & \multirow[b]{2}{*}{ PSA (ng/mL) } & \multirow[b]{2}{*}{ PSA range } & \multirow[b]{2}{*}{ Design } & \multicolumn{2}{|c|}{ PET protocol } \\
\hline & & & & & & & & $\begin{array}{l}\text { Injected } \\
\text { activity }\end{array}$ & $\begin{array}{l}\text { Uptake } \\
\text { time (min) }\end{array}$ \\
\hline Afaq (18) & 2018 & 100 & $\mathrm{BCR}$ & 67.9 & NR & NR & $\mathrm{R}$ & $159 \mathrm{MBq}$ & 60 \\
\hline Afshar-Oromieh (19) & 2013 & 37 & $\mathrm{BCR}$ & 70 & 3.3 & $0.01-148$ & NR & $121.0 \mathrm{MBq}$ & 60 \\
\hline Afshar-Oromieh (3) & 2014 & 37 & BCR & 69.3 & 11.1 & $0.01-116$ & $\mathrm{R}$ & $132.0 \mathrm{MBq}$ & 60 \\
\hline Afshar-Oromieh (20) & 2014 & 20 & $\mathrm{BCR}$ & 69.6 & 2.62 & $0.51-73.60$ & NR & $149.0 \mathrm{MBq}$ & 60 \\
\hline Afshar-Oromieh (14) & 2015 & 319 & IS + BCR & 68 & 4.59 & $0.01-41395$ & $\mathrm{R}$ & $168 \mathrm{MBq}$ & 60 \\
\hline Afshar-Oromieh (13) & 2017 & 1007 & BCR & 68 & 12.1 & $0.01-1237$ & $\mathrm{R}$ & $227 \mathrm{MBq}$ & 68 \\
\hline Afshar-Oromieh (21) & 2017 & 112 & IS + BCR & 70 & 6.39 & $0.01-176.0$ & $\mathrm{R}$ & $207 \mathrm{MBq}$ & 60 \\
\hline Bluemel (22) & 2016 & 32 & $\mathrm{BCR}$ & 69 & 5.4 & $0.2-126.65$ & $\mathrm{R}$ & $133 \mathrm{MBq}$ & 60 \\
\hline Budaus (23) & 2016 & 30 & IS & 62 & 38.9 & $1.4-376$ & $\mathrm{R}$ & $169.4 \mathrm{MBq}$ & 60 \\
\hline Byrne (24) & 2018 & 81 & $\mathrm{BCR}$ & 63 & 0.87 & $0.22-8.7$ & NR & $2 \mathrm{MBq} / \mathrm{kg}$ & 60 \\
\hline Ceci (25) & 2015 & 70 & BCR & 67 & 3.5 & $0.2-32.2$ & $\mathrm{R}$ & $146.3 \mathrm{MBq}$ & 60 \\
\hline Demirkol (26) & 2015 & 22 & BCR & 68 & 4.15 & $0.2-191.5$ & $\mathrm{R}$ & $166.0 \mathrm{MBq}$ & 45 \\
\hline Eiber (1) & 2015 & 248 & BCR & 70 & 1.99 & $0.2-59.4$ & $\mathrm{R}$ & $155 \mathrm{MBq}$ & 54.2 \\
\hline Einspieler (27) & 2017 & 118 & BCR & 72 & 6.4 & $2.2-158.4$ & $\mathrm{R}$ & $2 \mathrm{MBq} / \mathrm{kg}$ & 60 \\
\hline Fendler (28) & 2016 & 21 & IS & NR & NR & NR & $\mathrm{R}$ & $192 \mathrm{MBq}$ & 58 \\
\hline Giesel (29) & 2015 & 21 & $\mathrm{BCR}$ & 70 & 6.84 & $0.6-45$ & NR & $176 \mathrm{MBq}$ & 60 \\
\hline Grubmüller (30) & 2018 & 117 & BCR & 74 & 1.04 & $0.58-1.87$ & $\mathrm{R}$ & $2 \mathrm{MBq} / \mathrm{kg}$ & 60 \\
\hline Gupta (12) & 2017 & 179 & $\mathrm{BCR}$ & 70 & 4.7 & $0.01-963$ & $\mathrm{R}$ & $149 \mathrm{MBq}$ & 50 \\
\hline Habl (31) & 2017 & 100 & BCR & 64 & 1 & $0.12-14.7$ & $\mathrm{R}$ & $146 \mathrm{MBq}$ & 56 \\
\hline Henkenberens (32) & 2017 & 23 & BCR & 80 & 2.75 & $0.52-8.92$ & $\mathrm{R}$ & $79 \mathrm{MBq}$ & 60 \\
\hline Herlemann (33) & 2016 & 34 & IS + BCR & NR & NR & NR & $\mathrm{R}$ & NR & 60 \\
\hline Herlemann (34) & 2017 & 35 & $\mathrm{BCR}$ & 64 & 4.1 & NR & $\mathrm{R}$ & NR & NR \\
\hline Hope (10) & 2017 & 126 & BCR & 69 & 5.9 & NR & PR & $199.8 \mathrm{MBq}$ & 63 \\
\hline Hruby (35) & 2017 & 48 & BCR & NR & 5 & $2.04-39$ & NR & $2.0 \mathrm{MBq} / \mathrm{kg}$ & 60 \\
\hline Kranzbuhler (9) & 2018 & 56 & BCR & 69 & 0.99 & $0.05-30$ & $\mathrm{R}$ & $123 \mathrm{MBq}$ & 60 \\
\hline Lake (36) & 2017 & 55 & $\mathrm{BCR}$ & 68.3 & 11.2 & $4-88$ & $\mathrm{R}$ & $201.5 \mathrm{MBq}$ & 65 \\
\hline Maurer (37) & 2016 & 130 & IS & 66 & 11.5 & $6.85-24.5$ & $\mathrm{R}$ & $1.76 \mathrm{MBq} / \mathrm{kg}$ & 59.8 \\
\hline Morigi (2) & 2015 & 38 & BCR & 68 & 15.6 & $0.04-12.0$ & PR & $2 \mathrm{MBq} / \mathrm{kg}$ & 60 \\
\hline Pfister (38) & 2016 & 28 & $\mathrm{BCR}$ & 67 & 2.35 & $0.04-8$ & $\mathrm{R}$ & $2 \mathrm{MBq} / \mathrm{kg}$ & 45 \\
\hline Rauscher (39) & 2016 & 48 & BCR & 71 & 1.31 & $0.75-2.55$ & $\mathrm{R}$ & $154 \mathrm{MBq}$ & 57 \\
\hline Rauscher (40) & 2016 & 22 & BCR & 68 & 1.03 & $0.2-.72$ & $\mathrm{R}$ & $147 \mathrm{MBq}$ & 60 \\
\hline Sachpekidis (41) & 2016 & 31 & $\mathrm{BCR}$ & 71 & 2 & $0.1-130$ & NR & $236 \mathrm{MBq}$ & 60 \\
\hline Sanli (42) & 2017 & 109 & BCR & 71 & 6.5 & $0.2-640$ & $\mathrm{R}$ & $185 \mathrm{MBq}$ & 60 \\
\hline Schiller (43) & 2017 & 31 & BCR & 64 & 2.19 & $0.12-14.7$ & $\mathrm{R}$ & $135 \mathrm{MBq}$ & 60 \\
\hline Schmidt-Hegemann (44) & 2017 & 129 & BCR & 72 & 6.04 & $0.13-150.00$ & $\mathrm{R}$ & $189 \mathrm{MBq}$ & 60 \\
\hline Siriwardana (45) & 2017 & 35 & $\mathrm{BCR}$ & 67 & 0.2 & $0-1$ & $\mathrm{R}$ & NR & NR \\
\hline Uprimny (46) & 2017 & 203 & $\mathrm{BCR}$ & 68 & 1.44 & $0.14-96.0$ & $\mathrm{R}$ & $150 \mathrm{MBq}$ & 60 \\
\hline Van Leeuwen (11) & 2016 & 70 & BCR & 67 & 0.2 & $0.12-.32$ & PR & NR & NR \\
\hline Van Leeuwen (47) & 2017 & 30 & IS & 65 & 8.1 & $5.2-10.1$ & PR & NR & 60 \\
\hline Verburg (48) & 2016 & 155 & BCR & 70 & 4 & $0-2,000$ & $\mathrm{R}$ & $190 \mathrm{MBq}$ & 60 \\
\hline Zhang (49) & 2017 & 42 & IS + BCR & 69 & 52.31 & $7.20-348$ & $\mathrm{R}$ & $131.7 \mathrm{MBq}$ & 60 \\
\hline
\end{tabular}

$\mathrm{BCR}=$ biochemical recurrence; IS = initial staging; $\mathrm{R}=$ retrospective; $\mathrm{PR}=$ prospective; $\mathrm{NR}=$ not reported.

Means are provided when available; otherwise, medians reported. 
TABLE 2

Overview of Included Initial Staging in 5 Studies Reporting Total of 266 Patients

\begin{tabular}{|c|c|c|c|c|c|c|c|}
\hline Study & Year & $\begin{array}{l}\text { D'Amico } \\
\text { risk }\end{array}$ & $n$ & $\begin{array}{l}\text { Nodes/ } \\
\text { patient }\end{array}$ & TP FP & P TN & FN \\
\hline Budaus (23) & 2016 & $\mathrm{~N}$ & 30 & 20.3 & 40 & 18 & $\varepsilon$ \\
\hline $\begin{array}{l}\text { Herlemann } \\
\text { (33) }\end{array}$ & 2016 & $\mathrm{~N}$ & 34 & 14.2 & 204 & 48 & 2 \\
\hline Maurer (37) & 2016 & $Y$ & 130 & $21^{*}$ & 271 & 188 & 14 \\
\hline $\begin{array}{l}\text { Van Leeuwen } \\
\text { (47) }\end{array}$ & 2017 & Y & 30 & 17.8 & 71 & 118 & 4 \\
\hline Zhang (49) & 2017 & Y & 42 & 14.8 & 141 & 126 & 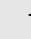 \\
\hline
\end{tabular}

${ }^{*}$ Reported as median.

$\mathrm{TP}=$ true positive; FP = false positive; $\mathrm{TN}=$ true negative; $\mathrm{FN}=$ false negative.

Number of nodes resected per patient is reported as mean. Papers that use intermediate to high risk by D'Amico classification for inclusion criteria are noted.

accuracy in all patients with pathology correlation were 0.99 (95\% CI, 0.96-1.00), 0.76 (95\% CI, 0.02-1.00), 0.99 (95\% CI, 0.96-1.00), 0.76 (95\% CI, 0.02-1.00), and 0.98 (95\% CI, 0.94$1.00)$, respectively. Given that only PSMA-positive lesions were biopsied and the resultant low number of true- and false-negative lesions, the most relevant measurement in this population is the PPV (Fig. 2).

The reporting of detection rate was heterogeneous across PSA levels, and comparisons across all papers is limited within specific

TABLE 3

Overview of Included Biochemical Recurrence Studies Reporting 256 Patients in Total with Pathology Correlation

\begin{tabular}{|lrrrrrr}
\hline \multicolumn{1}{c}{ Study } & Year & $n$ & TP & FP & TN & FN \\
\hline Afaq (18) & 2018 & 11 & 10 & 1 & 0 & 0 \\
\hline Afshar-Oromieh (19) & 2013 & 6 & 6 & 0 & 0 & 0 \\
\hline Afshar-Oromieh (3) & 2014 & 7 & 7 & 0 & 0 & 0 \\
\hline Afshar-Oromieh (14) & 2015 & 42 & 37 & 0 & 0 & 5 \\
\hline Ceci (25) & 2015 & 7 & 6 & 1 & 0 & 0 \\
\hline Demirkol (26) & 2015 & 3 & 3 & 0 & 0 & 0 \\
\hline Einspieler (27) & 2017 & 6 & 6 & 0 & 0 & 0 \\
\hline Morigi (2) & 2015 & 10 & 9 & 0 & 1 & 0 \\
\hline Eiber (1) & 2015 & 12 & 12 & 0 & 0 & 0 \\
\hline Grubmüller (30) & 2018 & 16 & 16 & 0 & 0 & 0 \\
\hline Pfister (38) & 2016 & 28 & 22 & 6 & 0 & 0 \\
\hline Rauscher (39) & 2016 & 22 & 22 & 0 & 0 & 0 \\
\hline Rauscher (50) & 2016 & 48 & 42 & 3 & 3 & 0 \\
\hline Siriwardana (45) & 2017 & 35 & 32 & 0 & 3 & 0 \\
\hline Van Leeuwen (11) & 2016 & 3 & 3 & 0 & 0 & 0 \\
\hline
\end{tabular}

$\mathrm{TP}=$ true positive; $\mathrm{FP}=$ false positive; $\mathrm{TN}=$ true negative; $\mathrm{FN}=$ false negative.

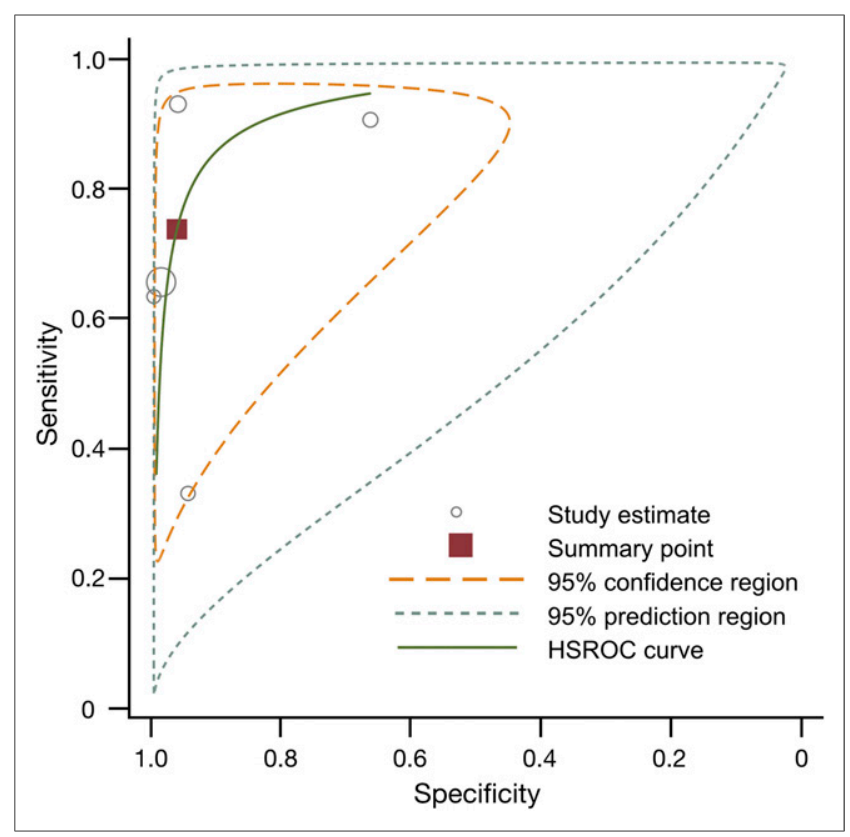

FIGURE 2. Summary of sensitivity, specificity, and hierarchical summary receiver-operating-characteristic (HSROC) curve for ${ }^{68} \mathrm{Ga}-\mathrm{PSMA}-$ 11 for initial staging of intermediate- to high-risk prostate cancer patients before prostatectomy for malignancy in pelvic nodes, with pathology at time of prostatectomy as gold standard. Effect size for sensitivity and specificity was $0.74(95 \% \mathrm{Cl}, 0.51-0.89)$ and $0.96(95 \% \mathrm{Cl}, 0.85-0.99)$, respectively. Size of circles represents size of individual studies.

PSA ranges. We grouped papers that reported results with a PSA of less than $2.0 \mathrm{ng} / \mathrm{dL}$, between 2.0 and $5.0 \mathrm{ng} / \mathrm{dL}$, and more than $5.0 \mathrm{ng} / \mathrm{dL}$ (Table 4). The metaanalysis for the detection rate for PSAs of less than 2.0 was $0.6395 \%$ CI, (0.55-0.70). For PSAs of 2.0-5.0 the estimate was 0.89 (95\% CI, 0.85-0.93) (Fig. 4), and for PSAs of more than 5.0 the estimate was 0.95 (95\% CI, 0.92-0.97). We grouped papers that reported detection rates above and below PSAs of $2.0 \mathrm{ng} / \mathrm{mL}$, and we provide a Forest plot of the results (Fig. 3).

Six papers reported detection sensitivity in patients with a PSA of less than $0.2 \mathrm{ng} / \mathrm{mL}$, although few data were reported on pathology correlation in these papers (9-14). In these papers, 61 of 153 patients were reported as being positive for disease on PSMA PET, and the metaanalysis for the detection rate for PSAs of less than 0.2 was 0.4 (95\% CI, 0.24-0.57). The largest study reported 32 of 68 positive for disease when the PSA level was less than $0.2 \mathrm{nd} / \mathrm{dL}(13)$.

\section{DISCUSSION}

This metaanalysis reaffirms the utility of ${ }^{68} \mathrm{Ga}$-PSMA-11 PET for imaging of intermediate- to high-risk patients before definitive therapy and those who develop subsequent biochemical recurrence. In initial staging with pathology as a gold standard, ${ }^{68} \mathrm{Ga}$-PSMA-11 had a sensitivity and specificity of 0.74 (95\% CI, $0.51-0.89)$ and 0.96 (95\% CI, 0.85-0.99), respectively, and in biochemical recurrence, the PPV was 0.99 (95\% CI, 0.96-1.00). For those patients with biochemical recurrence, the detection rate was $0.63(95 \% \mathrm{CI}$, 0.55-0.70) with a PSA of less than 2.0 and 0.94 (95\% CI, 0.910.96 ) with a PSA of more than 2.0 .

The sensitivity and specificity results in our study can be difficult to compare with other studies. Perera et al. reported a sensitivity and 
TABLE 4

Overview of Detection Sensitivity for ${ }^{68}$ Ga-PSMA-11 PET Stratified by PSA Level at Time of Imaging in 2,616 Patients

\begin{tabular}{|c|c|c|c|c|c|c|c|c|}
\hline \multirow[b]{2}{*}{ Study } & \multirow[b]{2}{*}{ Year } & \multirow[b]{2}{*}{$n$} & \multicolumn{3}{|c|}{ Positive patients/total } & \multicolumn{3}{|c|}{ Detection rate by PSA } \\
\hline & & & $<2$ & $2-5$ & $>5$ & $<2$ & $2-5$ & $>5$ \\
\hline Afshar-Oromieh (19) & 2013 & 37 & $9 / 10$ & $9 / 13$ & $14 / 14$ & $90 \%$ & $69 \%$ & $100 \%$ \\
\hline Afshar-Oromieh (14) & 2015 & 311 & $55 / 90$ & $64 / 73$ & $140 / 148$ & $61 \%$ & $88 \%$ & $95 \%$ \\
\hline Afshar-Oromieh (13) & 2017 & 960 & $301 / 451$ & $201 / 227$ & $266 / 282$ & $67 \%$ & $89 \%$ & $94 \%$ \\
\hline Bluemel (22) & 2016 & 32 & $9 / 25$ & $5 / 7$ & & $36 \%$ & $71 \%$ & \\
\hline Ceci (25) & 2015 & 51 & $17 / 20$ & $29 / 31$ & & $85 \%$ & $94 \%$ & \\
\hline Demirkol (26) & 2015 & 22 & $5 / 7$ & $15 / 15$ & & $71 \%$ & $100 \%$ & \\
\hline Eiber (1) & 2015 & 248 & $102 / 124$ & $120 / 124$ & & $82 \%$ & $97 \%$ & \\
\hline Einspieler (27) & 2017 & 118 & NR & $36 / 44$ & $71 / 74$ & NR & $82 \%$ & $96 \%$ \\
\hline Gupta (12) & 2017 & 177 & $24 / 56$ & $117 / 121$ & & $43 \%$ & $97 \%$ & \\
\hline Habl (31) & 2017 & 100 & $56 / 80$ & $20 / 20$ & & $70 \%$ & $100 \%$ & \\
\hline Kranzbuhler (9) & 2018 & 56 & $24 / 35$ & $20 / 21$ & & $69 \%$ & $95 \%$ & \\
\hline Lake (36) & 2017 & 55 & $14 / 18$ & $35 / 37$ & & $78 \%$ & $95 \%$ & \\
\hline Hope (10) & 2017 & 121 & $41 / 55$ & $20 / 21$ & $42 / 45$ & $75 \%$ & $95 \%$ & $93 \%$ \\
\hline Morigi (2) & 2015 & 38 & $18 / 30$ & $7 / 8$ & & $60 \%$ & $88 \%$ & \\
\hline Sachpekidis (41) & 2016 & 31 & $7 / 15$ & $12 / 16$ & & $47 \%$ & $75 \%$ & \\
\hline Sanli (42) & 2017 & 25 & $5 / 16$ & $9 / 9$ & NR & $31 \%$ & $100 \%$ & NR \\
\hline Van Leeuwen (11) & 2016 & 70 & $25 / 70$ & NR & NR & $36 \%$ & NR & NR \\
\hline Verburg (48) & 2016 & 155 & $27 / 46$ & $97 / 109$ & & $59 \%$ & $89 \%$ & \\
\hline $\begin{array}{l}\mathrm{PSA} \text { is in } \mathrm{ng} / \mathrm{mL} \text {. } \\
\mathrm{NR}=\text { not reported. }\end{array}$ & & & & & & & & \\
\hline
\end{tabular}

specificity of 0.86 (95\% CI, 0.37-0.98) and 0.86 (95\% CI, $0.03-$ 1.00), respectively, but merged data from both biochemical recurrence and initial staging patients (5). A second, more recent, metaanalysis

\begin{tabular}{|c|c|c|c|}
\hline Study & & ES (95\% Cl) & $\%$ Weight \\
\hline Afshar-Oromieh (2013) & & $\longrightarrow 1.00(0.61,1.00)$ & 3.46 \\
\hline Afshar-Oromieh (2014) & & $\longrightarrow 1.00(0.65,1.00)$ & 3.92 \\
\hline Afshar-Oromieh (2015) & & $\longrightarrow 1.00(0.91,1.00)$ & 12.48 \\
\hline Ceci (2015) & & $\begin{array}{ll}- & 0.86(0.49,0.97)\end{array}$ & 3.92 \\
\hline Demirkol (2015) & & $\stackrel{1.00(0.44,1.00)}{\longrightarrow}$ & 1.98 \\
\hline Eiber (2015) & & $\longrightarrow 1.00(0.76,1.00)$ & 5.96 \\
\hline Morigi (2015) & & $\longrightarrow 1.00(0.70,1.00)$ & 4.78 \\
\hline Pfister (2016) & & $0.79(0.60,0.90)$ & 10.65 \\
\hline Rauscher (2016) & & $\longrightarrow 1.00(0.85,1.00)$ & 9.15 \\
\hline Rauscher (2016) & & $\longrightarrow \begin{array}{l:l}\longrightarrow \rightarrow & 0.93(0.82,0.98)\end{array}$ & 13.81 \\
\hline Van Leeuwen (2016) & & $\longrightarrow 1.00(0.44,1.00)$ & 1.98 \\
\hline Einspieler (2017) & & $\longrightarrow 1.00(0.61,1.00)$ & 3.46 \\
\hline Grubmüller (2017) & & $\longrightarrow 1.00(0.81,1.00)$ & 7.36 \\
\hline Siriwardana (2017) & & $\longrightarrow 1.00(0.89,1.00)$ & 11.52 \\
\hline Afaq (2018) & & 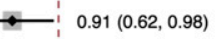 & 5.58 \\
\hline Overall $\left(\left.\right|^{\wedge 2}=26.18 \%, P=0.17\right)$ & & $\Delta 0.99(0.96,1.00)$ & 100.00 \\
\hline $\begin{array}{l}1 \\
0\end{array}$ & $\begin{array}{c}1 \\
0.5\end{array}$ & $\begin{array}{c}1 \\
1.0\end{array}$ & \\
\hline
\end{tabular}

FIGURE 3. Forest plot of PPV for ${ }^{68} \mathrm{Ga}-\mathrm{PSMA}-11$ in biochemical recurrence patients who have pathologic correlation for gold standard comparison. Overall effect size (ES) for PPV is $0.99(95 \% \mathrm{Cl}$, 0.96-1.00). Size of squares represents size of individual studies. Reference numbers are in Table 3. performed only on the initial staging population closely mirrored our results, with estimated sensitivity and specificity of $0.71(95 \%$ CI, 0.59-0.81) and 0.95 (95\% CI, 0.87-0.99), respectively (15). von Eyben et al. reported the sensitivity and specificity for initial staging alone- 0.70 (95\% CI, 0.53-0.83) —and pooled specificity was 0.84 (95\% CI, 24-99) (4). The point estimates from von Eyben more closely mirror our results, although our confidence intervals are narrower because of a greater number of patients published since the von Eyben article. von Eyben et al. did not perform a metaanalysis compared with histopathology in the biochemical recurrence setting.

In biochemical recurrence, we chose to report a PPV and not the sensitivity and specificity, as only PSMA-avid lesions are typically biopsied and therefore the subsequent calculated sensitivity and specificity are not relevant. Given that all patients with biochemical recurrence are considered to have disease, the detection rates may be used to approximate the sensitivity for metastatic disease in these patients. It is not possible to biopsy numerous nodes in patients, and therefore the accuracy of ${ }^{68} \mathrm{Ga}$ PSMA-11 PET outside biopsied lesions is unknown. It is possible that ${ }^{68} \mathrm{Ga}$-PSMA-11 


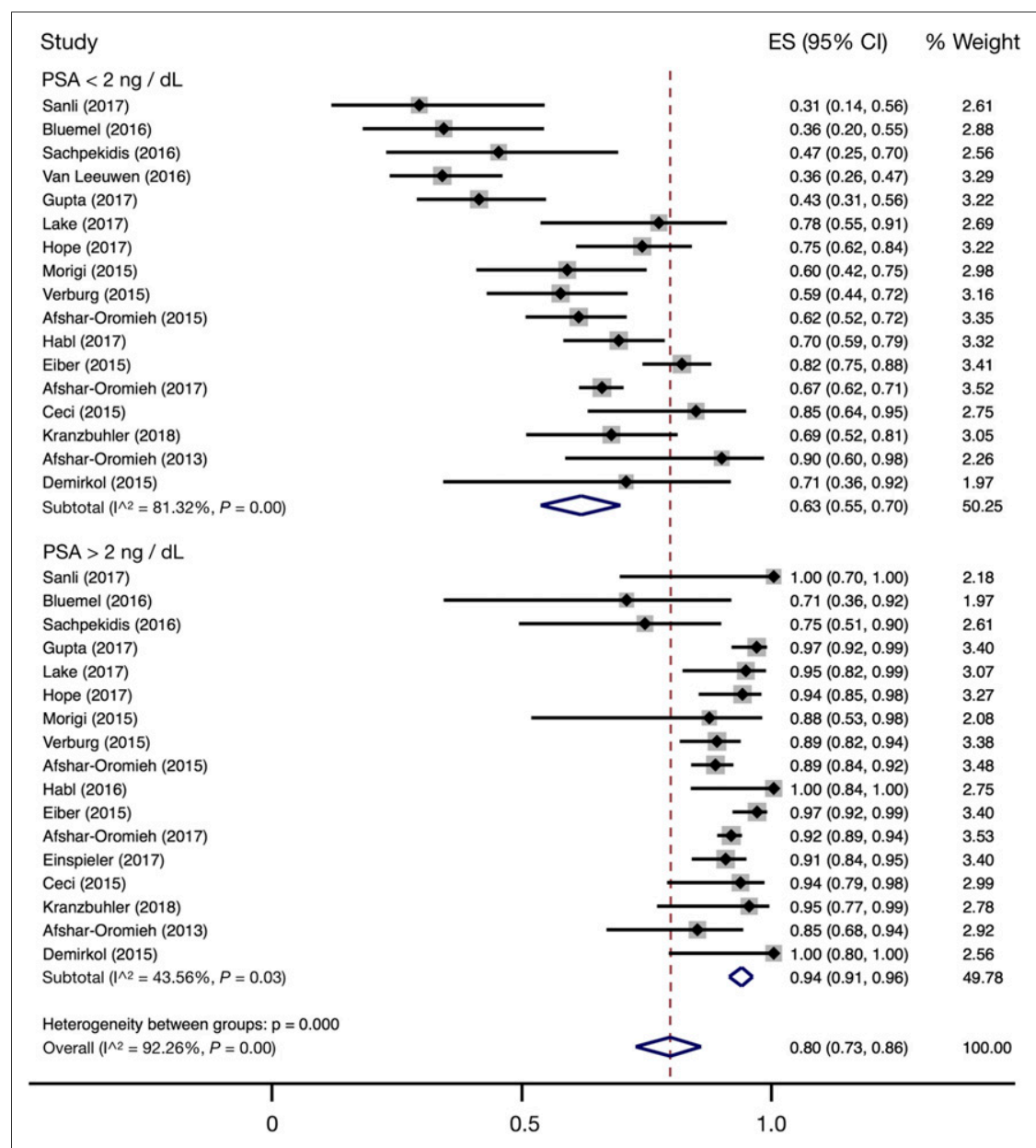

FIGURE 4. Forest plot of detection rate for ${ }^{68} \mathrm{Ga}-\mathrm{PSMA}-11$ in biochemical recurrence patients. Effect size (ES) for detection rate is $0.63(95 \% \mathrm{Cl}, 0.55-0.70)$ for $\mathrm{PSA}<2.0 \mathrm{ng} / \mathrm{dL}$ and $0.94(95 \%$ $\mathrm{Cl}, 0.91-0.96)$ for PSA $>2.0 \mathrm{ng} / \mathrm{dL}$. Size of squares represents size of individual studies. Reference numbers are in Table 3.

PET sees only the tip of the iceberg in a large number of patients and that there may be several negative lesions that are not detected and not biopsied.

We chose to limit our analysis to lesions that have a biopsy correlate, because the definition of reference standards varied across the papers that used a composite endpoint of clinical and imaging follow-up. One of the main reasons for the lack of pathology correlation is the difficulty in obtaining a biopsy of lesions in patients with biochemical recurrence at low PSAs. The absence of gold-standard verification makes measurements of accuracy in this population difficult. Given that there is no agreed-upon follow-up composite gold standard, it is not possible to pool data from patients who do not have pathologic validation. Nonetheless, there was a relatively large number of patients (256) reported in the literature with pathologic correlation.

When reporting the results of research radiopharmaceuticals, it is important to consider how the data included may be used to support the subsequent radiopharmaceutical approval. In some cases, literature-based metaanalysis can be used in lieu of a second registration trial, and therefore the quality of studies is critical in support of future approvals. There are several things that are frequently not reported, including safety, inclusion and exclusion criteria, radiopharmaceutical synthesis methods, and quality control method. Using multiple readers and reporting interreader variability is also important in strengthening the value of the results. Dose ranges and uptake times are frequently reported by what is defined in the imaging protocol and not what occurred in individual patients. Another factor that is frequently underdescribed in the literature is the chemistry process (source of the precursor, synthesis module used, generator type, and quality assurance process used), which are important in the registration process so that regulatory bodies know that identical compounds were used across studies. There may be value in developing standard reporting guidelines for studies that evaluate the role of radiopharmaceuticals to ensure high-quality data in the literature moving forward. Furthermore, there may be value in developing harmonized release criteria across sites using the same compound in the research setting to help keep radiopharmaceutical products consistent across sites.

In addition to improved harmonization of reporting in PSMA PET articles, what would greatly strengthen the results in the literature are well-designed prospective studies that include a well-defined gold standard that can be used to measure accuracy. Although this is an optimistic goal, we readily admit that in the setting of biochemical recurrence, this goal may be difficult to achieve given the general frequent inability to obtain histologic verification.

The main limitation of our study is the heterogeneity that exists within the included studies. For example, patients are grouped by varying PSA ranges in the literature, which makes determining the reported sensitivities within various PSA ranges difficult to pool across articles. Additionally, most studies assessing imaging in those with biochemical recurrence did not include pathology correlation and simply reported detection sensitivities. Finally, the interpretive criteria are not defined in all papers, and in the past year reporting standards have been proposed that may limit variability in readings $(16,17)$. One of the main reasons for the heterogeneity across studies using ${ }^{68} \mathrm{Ga}-$ PSMA-11 is that most reports are from institutions using the compound under a compassionate-use setting, and so no formal prospective protocols were developed or followed.

\section{CONCLUSION}

${ }^{68} \mathrm{Ga}$-PSMA-11 performed well for the localization of metastatic prostate cancer. In initial staging, with pathology as a gold standard, ${ }^{68} \mathrm{Ga}$-PSMA-11 had a sensitivity and specificity of 0.74 (95\% CI, 0.51-0.89) and 0.96 (95\% CI, 0.85-0.99). In biochemical recurrence, with pathology as a gold standard, the PPV was 0.99 (95\% CI, 0.96-1.00). The detection rate was 0.63 (95\% CI, 
0.55-0.70) with a PSA less than 2.0 and 0.94 (95\% CI, 0.91-0.96) with a PSA greater than 2.0.

\section{DISCLOSURE}

Thomas Hope receives grant support from the 2017 Jonathan Kovler Young Investigator Award from the Prostate Cancer Foundation, and the National Institutes of Health (R01CA212148). No other potential conflict of interest relevant to this article was reported.

\section{REFERENCES}

1. Eiber M, Maurer T, Souvatzoglou M, et al. Evaluation of hybrid ${ }^{68} \mathrm{Ga}-\mathrm{PSMA}$ ligand PET/CT in 248 patients with biochemical recurrence after radical prostatectomy. J Nucl Med. 2015;56:668-674.

2. Morigi JJ, Stricker PD, van Leeuwen PJ, et al. Prospective comparison of ${ }^{18} \mathrm{~F}-$ fluoromethylcholine versus ${ }^{68} \mathrm{Ga}$-PSMA PET/CT in prostate cancer patients who have rising PSA after curative treatment and are being considered for targeted therapy. J Nucl Med. 2015;56:1185-1190.

3. Afshar-Oromieh A, Zechmann CM, Malcher A, et al. Comparison of PET imaging with a ${ }^{68} \mathrm{Ga}$-labelled PSMA ligand and ${ }^{18} \mathrm{~F}$-choline-based PET/CT for the diagnosis of recurrent prostate cancer. Eur J Nucl Med Mol Imaging. 2014;41: 11-20.

4. von Eyben FE, Picchio M, von Eyben R, Rhee H, Bauman G. ${ }^{68}$ Ga-labeled prostate-specific membrane antigen ligand positron emission tomography/computed tomography for prostate cancer: a systematic review and meta-analysis. Eur Urol Focus. 2018;4:686-693.

5. Perera M, Papa N, Christidis D, et al. Sensitivity, specificity, and predictors of positive ${ }^{68} \mathrm{Ga}$-prostate-specific membrane antigen positron emission tomography in advanced prostate cancer: a systematic review and meta-analysis. Eur Urol. 2016;70:926-937.

6. Moher D, Liberati A, Tetzlaff J, Altman DG; PRISMA Group. Preferred reporting items for systematic reviews and meta-analyses: the PRISMA statement. PLoS Med. 2009;6:e1000097.

7. Whiting PF, Rutjes AWS, Westwood ME, et al. QUADAS-2: a revised tool for the quality assessment of diagnostic accuracy studies. Ann Intern Med. 2011;155: 529-536.

8. DerSimonian R, Laird N. Meta-analysis in clinical trials. Control Clin Trials. 1986;7:177-188.

9. Kranzbühler B, Nagel H, Becker AS, et al. Clinical performance of ${ }^{68}$ Ga-PSMA$11 \mathrm{PET} / \mathrm{MRI}$ for the detection of recurrent prostate cancer following radical prostatectomy. Eur J Nucl Med Mol Imaging. 2018;45:20-30.

10. Hope TA, Aggarwal R, Chee B, et al. Impact of ${ }^{68} \mathrm{Ga}$-PSMA-11 PET on management in patients with biochemically recurrent prostate cancer. $\mathrm{J} \mathrm{Nucl} \mathrm{Med}$. 2017;58:1956-1961.

11. van Leeuwen PJ, Stricker P, Hruby G, et al. ${ }^{68}$ Ga-PSMA has a high detection rate of prostate cancer recurrence outside the prostatic fossa in patients being considered for salvage radiation treatment. BJU Int. 2016;117:732-739.

12. Gupta SK, Watson T, Denham J, et al. Prostate-specific membrane antigen positron emission tomography-computed tomography for prostate cancer: distribution of disease and implications for radiation therapy planning. Int $J$ Radiat Oncol Biol Phys. 2017;99:701-709.

13. Afshar-Oromieh A, Holland-Letz T, Giesel FL, et al. Diagnostic performance of ${ }^{68} \mathrm{Ga}$-PSMA-11 (HBED-CC) PET/CT in patients with recurrent prostate cancer: evaluation in 1007 patients. Eur J Nucl Med Mol Imaging. 2017;44: $1258-1268$.

14. Afshar-Oromieh A, Avtzi E, Giesel FL, et al. The diagnostic value of PET/ $\mathrm{CT}$ imaging with the ${ }^{68} \mathrm{Ga}$-labelled PSMA ligand HBED-CC in the diagnosis of recurrent prostate cancer. Eur J Nucl Med Mol Imaging. 2015;42:197-209.

15. Kim S-J, Lee S-W, Ha HK. Diagnostic performance of radiolabeled prostatespecific membrane antigen positron emission tomography/computed tomography for primary lymph node staging in newly diagnosed intermediate to high-risk prostate cancer patients: a systematic review and meta-analysis. Urol Int. 2019;102: 27-36.

16. Eiber M, Herrmann K, Calais J, et al. Prostate cancer molecular imaging standardized evaluation (PROMISE): proposed miTNM classification for the interpretation of PSMA-ligand PET/CT. J Nucl Med. 2018;59:469-478.

17. Rowe SP, Pienta KJ, Pomper MG, Gorin MA. Proposal for a structured reporting system for prostate-specific membrane antigen-targeted PET imaging: PSMARADS version 1.0. J Nucl Med.2018;59:479-485.
18. Afaq A, Alahmed S, Chen S-H, et al. Impact of ${ }^{68} \mathrm{Ga}$-prostate-specific membrane antigen PET/CT on prostate cancer management. J Nucl Med. 2018;59:89-92.

19. Afshar-Oromieh A, Malcher A, Eder M, et al. PET imaging with a $\left[{ }^{68} \mathrm{Ga}\right]$ gallium-labelled PSMA ligand for the diagnosis of prostate cancer: biodistribution in humans and first evaluation of tumour lesions. Eur J Nucl Med Mol Imaging. 2013;40:486-495

20. Afshar-Oromieh A, Haberkorn U, Schlemmer HP, et al. Comparison of PET/CT and PET/MRI hybrid systems using a ${ }^{68} \mathrm{Ga}$-labelled PSMA ligand for the diagnosis of recurrent prostate cancer: initial experience. Eur J Nucl Med Mol Imaging. 2014;41:887-897.

21. Afshar-Oromieh A, Sattler LP, Mier W, et al. The clinical impact of additional late PET/CT imaging with ${ }^{68} \mathrm{Ga}$-PSMA-11 (HBED-CC) in the diagnosis of prostate cancer. J Nucl Med. 2017;58:750-755.

22. Bluemel C, Krebs M, Polat B, et al. ${ }^{68} \mathrm{Ga}-\mathrm{PSMA}-\mathrm{PET} / \mathrm{CT}$ in patients with biochemical prostate cancer recurrence and negative ${ }^{18} \mathrm{~F}$-choline-PET/CT. Clin Nucl Med. 2016;41:515-521.

23. Budäus L, Leyh-Bannurah S-R, Salomon G, et al. Initial experience of ${ }^{68} \mathrm{Ga}-$ PSMA PET/CT imaging in high-risk prostate cancer patients prior to radical prostatectomy. Eur Urol. 2016;69:393-396.

24. Byrne K, Eade T, Kneebone A, et al. Delineating sites of failure following postprostatectomy radiation treatment using ${ }^{68} \mathrm{Ga}$-PSMA-PET. Radiother Oncol. 2018;126:244-248.

25. Ceci F, Uprimny C, Nilica B, et al. ${ }^{68}$ Ga-PSMA PET/CT for restaging recurrent prostate cancer: which factors are associated with PET/CT detection rate? Eur J Nucl Med Mol Imaging. 2015;42:1284-1294.

26. Demirkol MO, Acar Ö, Uçar B, Ramazanoğlu SR, Sağlıcan Y, Esen T. Prostatespecific membrane antigen-based imaging in prostate cancer: impact on clinical decision making process. Prostate. 2015;75:748-757.

27. Einspieler I, Rauscher I, Düwel C, et al. Detection efficacy of hybrid ${ }^{68} \mathrm{Ga}$-PSMA ligand PET/CT in prostate cancer patients with biochemical recurrence after primary radiation therapy defined by Phoenix criteria. J Nucl Med. 2017;58: 1081-1087.

28. Fendler WP, Schmidt DF, Wenter V, et al. ${ }^{68}$ Ga-PSMA PET/CT detects the location and extent of primary prostate cancer. J Nucl Med. 2016;57:17201725.

29. Giesel FL, Fiedler H, Stefanova M, et al. PSMA PET/CT with Glu-urea-Lys(Ahx)-[ ${ }^{68} \mathrm{Ga}($ HBED-CC)] versus 3D CT volumetric lymph node assessment in recurrent prostate cancer. Eur J Nucl Med Mol Imaging. 2015;42:17941800 .

30. Grubmüller B, Baltzer P, D'Andrea D, et al. ${ }^{68}$ Ga-PSMA 11 ligand PET imaging in patients with biochemical recurrence after radical prostatectomy: diagnostic performance and impact on therapeutic decision-making. Eur J Nucl Med Mol Imaging. 2018;45:235-242.

31. Habl G, Sauter K, Schiller K, et al. ${ }^{68}$ Ga-PSMA-PET for radiation treatment planning in prostate cancer recurrences after surgery: individualized medicine or new standard in salvage treatment. Prostate. 2017;77:920927.

32. Henkenberens C, VON Klot CA, Ross TL, et al. ${ }^{68}$ Ga-PSMA ligand PET/CTbased radiotherapy for lymph node relapse of prostate cancer after primary therapy delays initiation of systemic therapy. Anticancer Res. 2017;37:12731279 .

33. Herlemann A, Wenter V, Kretschmer A, et al. ${ }^{68}$ Ga-PSMA positron emission tomography/computed tomography provides accurate staging of lymph node regions prior to lymph node dissection in patients with prostate cancer. Eur Urol. 2016;70:553-557.

34. Herlemann A, Kretschmer A, Buchner A, et al. Salvage lymph node dissection after ${ }^{68} \mathrm{Ga}$-PSMA or ${ }^{18} \mathrm{~F}$-FEC PET/CT for nodal recurrence in prostate cancer patients. Oncotarget.2017;8:84180-84192.

35. Hruby G, Eade T, Kneebone A, et al. Delineating biochemical failure with ${ }^{68} \mathrm{Ga}-$ PSMA-PET following definitive external beam radiation treatment for prostate cancer. Radiother Oncol. 2017;122:99-102.

36. Lake ST, Greene KL, Westphalen AC, et al. Optimal MRI sequences for ${ }^{68} \mathrm{Ga}-$ PSMA-11 PET/MRI in evaluation of biochemically recurrent prostate cancer. EJNMMI Res. 2017;7:77.

37. Maurer T, Gschwend JE, Rauscher I, et al. Diagnostic efficacy of ${ }^{68}$ galliumPSMA positron emission tomography compared to conventional imaging for lymph node staging of 130 consecutive patients with intermediate to high risk prostate cancer. J Urol. 2016;195:1436-1443.

38. Pfister D, Porres D, Heidenreich A, et al. Detection of recurrent prostate cancer lesions before salvage lymphadenectomy is more accurate with ${ }^{68} \mathrm{Ga}-\mathrm{PSMA}-$ HBED-CC than with ${ }^{18}$ F-fluoroethylcholine PET/CT. Eur J Nucl Med Mol Imaging. 2016;43:1410-1417.

39. Rauscher I, Maurer T, Souvatzoglou M, et al. Intrapatient comparison of ${ }^{111}$ In-PSMA I\&T SPECT/CT and hybrid ${ }^{68} \mathrm{Ga}-\mathrm{HBED}-\mathrm{CC}$ PSMA PET in 
patients with early recurrent prostate cancer. Clin Nucl Med. 2016;41:e397e402.

40. Rauscher I, Maurer T, Beer AJ, et al. Value of ${ }^{68} \mathrm{Ga}$-PSMA HBED-CC PET for the assessment of lymph node metastases in prostate cancer patients with biochemical recurrence: comparison with histopathology after salvage lymphadenectomy. J Nucl Med. 2016;57:1713-1719.

41. Sachpekidis C, Eder M, Kopka K, et al. ${ }^{68}$ Ga-PSMA-11 dynamic PET/CT imaging in biochemical relapse of prostate cancer. Eur J Nucl Med Mol Imaging. 2016;43:1288-1299.

42. Sanli Y, Kuyumcu S, Sanli O, et al. Relationships between serum PSA levels, Gleason scores and results of ${ }^{68} \mathrm{Ga}$-PSMAPET/CT in patients with recurrent prostate cancer. Ann Nucl Med. 2017;31:709-717.

43. Schiller K, Sauter K, Dewes S, et al. Patterns of failure after radical prostatectomy in prostate cancer: implications for radiation therapy planning after ${ }^{68} \mathrm{Ga}-\mathrm{PSMA}$-PET imaging. Eur J Nucl Med Mol Imaging. 2017;44:1656-1662.

44. Schmidt-Hegemann N-S, Fendler WP, Buchner A, et al. Detection level and pattern of positive lesions using PSMA PET/CT for staging prior to radiation therapy. Radiat Oncol. 2017;12:176.

45. Siriwardana A, Thompson J, van Leeuwen PJ, et al. Initial multicentre experience of ${ }^{68}$ gallium-PSMA PET/CT guided robot-assisted salvage lymphadenectomy: acceptable safety profile but oncological benefit appears limited. BJU Int. 2017;120: 673-681.

46. Uprimny C, Kroiss AS, Decristoforo C, et al. Early dynamic imaging in ${ }^{68} \mathrm{Ga}$ - PSMA-11 PET/CT allows discrimination of urinary bladder activity and prostate cancer lesions. Eur J Nucl Med Mol Imaging. 2017;44:765775 .

47. van Leeuwen PJ, Emmett L, Ho B, et al. Prospective evaluation of ${ }^{68}$ Galliumprostate-specific membrane antigen positron emission tomography/computed tomography for preoperative lymph node staging in prostate cancer. BJU Int. 2017;119:209-215.

48. Verburg FA, Pfister D, Heidenreich A, et al. Extent of disease in recurrent prostate cancer determined by $\left[{ }^{68} \mathrm{Ga}\right] \mathrm{PSMA}-\mathrm{HBED}-\mathrm{CC}$ PET/CT in relation to PSA levels, PSA doubling time and Gleason score. Eur J Nucl Med Mol Imaging. 2016;43:397-403.

49. Zhang Q, Zang S, Zhang C, et al. Comparison of ${ }^{68}$ Ga-PSMA-11 PET-CT with mpMRI for preoperative lymph node staging in patients with intermediate to high-risk prostate cancer. J Transl Med. 2017;15:230.

50. Rauscher I, Maurer T, Fendler WP, Sommer WH, Schwaiger M, Eiber M. ${ }^{68} \mathrm{Ga}-$ PSMA ligand PET/CT in patients with prostate cancer: how we review and report. Cancer Imaging. 2016;16:14. 